\title{
NMR STUDIES OF
}

\section{MEMBRANE STRUCTURE AND DYNAMICS}

\author{
David F. Bocian and Sunney I. Chan \\ Arthur Amos Noyes Laboratory of Chemical Physics, California Institute of \\ Technology, Pasadena, California 91125
}

\section{INTRODUCTION}

Over the past decade, there has been considerable interest in the motional state of the phospholipid bilayer membrane. The motivation underlying these efforts has been the contention that the phospholipid bilayer is the basic matrix in which membrane proteins are embedded to form the biological membrane, and that the permeability and mechanical properties of the membrane, as well as the enzymatic activity of membrane proteins, are dependent upon the fluidity of the bilayer, especially the motional state of the hydrocarbon chains.

For many years, much of our knowledge about the motional state of the bilayer has come from Electron Spin Resonance (ESR) measurements on spin-labeled lipids incorporated into the bilayer $(1,2)$. Recent efforts have been directed toward developing spectroscopic probes that would circumvent the possible perturbations in the bilayer produced by the bulky nitroxide radical. Nuclear Magnetic Resonance (NMR) spectroscopy is a natural tool for this purpose and there has been considerable effort using ${ }^{1} \mathrm{H},{ }^{2} \mathrm{H},{ }^{13} \mathrm{C}$, and ${ }^{31} \mathrm{P}$ probes which are either endogenous or incorporated into the lipid molecule $(3-11)$. These efforts constitute the subject of the present review.

The application of NMR spectroscopy to the study of the lipid bilayer is not without some difficulties. In particular, the interpretation of NMR spectral parameters for a partially ordered system undergoing complex local motion is not straightforward, hence the conclusions deduced are often controversial because of the ambiguous way in which the data can be interpreted. These controversies are only now being resolved.

Because of the extensive coverage in the chemical literature given to NMR studies of phospholipid systems, no attempt is made to completely review this area of research. Instead, we concern ourselves with those investigations most pertinent to 


\section{8} BOCIAN \& CHAN

the determination of molecular order and the development of dynamical models for membrane systems. We first discuss the general consequences of order on the NMR spectra. Next we discuss specifically the evaluation of order parameters and their interpretation, the determination of acyl chain and head group dynamics, and lateral diffusion in membrane systems.

More general reviews of NMR studies of phospholipid systems can be found in the book by James (8), and in the articles by Lee et al (6), Chapman (3-5), and Podo (7). The review by Lee et al (6) contains a good general introduction to relaxation phenomena in lyotropic systems, but recent research has considerably modified the picture of the motional state of the lipid bilayer advanced by these workers. The comprehensive biannual reviews of NMR studies on liquid crystals by Tiddy $(10,11)$ also include extensive coverage of phospholipid systems. Finally, Wennerström \& Lindblom (9) have recently reviewed $I=\frac{1}{2}$ NMR studies of model membrane systems. This review contains an excellent general discussion of the mathematical formalism necessary for describing systems in which second-rank tensor interactions are incompletely averaged.

\section{THE PHOSPHOLIPID BILAYER}

Biological membranes are multicomponent systems composed of many different membrane proteins and lipids. Among the primary lipid constituents of most biological membranes are phospholipids, which occur naturally with a variety of head groups and acyl chains. Aqueous dispersions of phospholipids exhibit many of the physical properties of natural membranes, and accordingly, these dispersions have been used extensively as membrane models for NMR studies (3-11). Phospholipids spontaneously aggregate to form bilayer structures, and the interactions between different bilayers result in a thermodynamically stable multilamellar superstructure.

Pure phospholipid dispersions exist in a number of phases depending on temperature and water content (12). The two phases of primary importance as biological membrane models are the gel and the liquid crystalline phases. In multicomponent phospholipid systems, species immiscibility and phase separations can occur, and the phase diagrams become exceedingly complicated. For this reason, most NMR studies have been performed on single component systems, and, in general, at temperatures above the gel-liquid crystalline phase transition temperature. In principle, NMR could be used to study multicomponent systems, if the components could be suitably labeled.

Phospholipid multilayers are large units, typically with dimensions on the order of microns or greater. Thus, any motional averaging in the NMR spectra is determined by local molecular motions. In the gel phase, the local motions are significantly restricted and slow, with both inter- and intramolecular static dipolar interactions not significantly averaged on the NMR timescale $(6,8,9,13,14)$. This results in broad featureless NMR lines characteristic of organic solids with some internal motion. In contrast, in the liquid crystalline phase, local motions become less 
restricted and occur on a much faster timescale $(6,8,9,13,14)$. The result of this is an NMR spectrum with better resolved features, which is more interpretable.

As a general consequence of the restricted motion that occurs in phospholipid systems, all magnetic and electric second-rank tensor interactions are incompletely averaged, even in the liquid crystalline phase. This leads to first order effects which are manifested experimentally by 1 . broadening by nuclear dipolar interactions in the ${ }^{1} \mathrm{H}$ NMR spectra $(3-9,15-18), 2$. quadrupolar splittings in the ${ }^{2} \mathrm{H}$ NMR spectra (19-24), 3. broadening by nuclear dipolar interactions and by chemical shift anisotropy in the ${ }^{19} \mathrm{~F}$ spectra $(25,26)$, and 4 . broadening by chemical shift anisotropy in the proton-decoupled ${ }^{13} \mathrm{C}$ and ${ }^{31} \mathrm{P}$ spectra $(27-34)$. We now show how the extent of motional restriction, that is, molecular order, can be extracted from analysis of these first order effects.

\section{THE DETERMINATION OF MOLECULAR ORDER IN PHOSPHOLIPID SYSTEMS}

\section{The Order Parameter}

In motionally restricted systems it is customary to describe the extent of order with respect to a laboratory frame of reference. In general, this requires specifying a matrix of order parameters. Proton, deuterium, and phosphorus-31 NMR measurements on phospholipid dispersions oriented between glass plates show that, on a nuclear magnetic resonance timescale, effective axial symmetry exists about an axis normal to the bilayer surface in the liquid crystalline phase $(16,19,23,35)$. The off diagonal elements of the order parameter matrix are then zero when the molecular axes are referred to this symmetry axis, which is called the director. All motional information can be referred to this axis, and expressed in terms of the remaining order parameters, only two of which are independent. In the case of nuclear dipolar interactions and axially symmetric electric quadrupole interactions, only a single order parameter is extracted from the NMR experiment.

The order parameters for the methylene and methyl segments of multilamellar dispersions of phospholipids could be measured directly by ${ }^{1} \mathrm{H}$ NMR with selectively protonated perdeuterated lipids. In natural systems, however, the many-body interactions present complicate the determination of the segmental order parameters. Order parameters have been measured directly with ${ }^{2} \mathrm{H}$ NMR using selectively deuterated samples (19-24), but implicit in the ${ }^{2} \mathrm{H}$ NMR measurements is the assumption that the static quadrupolar interaction tensor for the C-D bond is axially symmetric. The segmental order parameters measured with ${ }^{2} \mathrm{H}$ NMR are, in general, different from those measured with ${ }^{1} \mathrm{H}$ NMR, since the deuterium quadrupolar interaction vector is directed along the C-D bond, whereas the protonproton dipolar interaction vector is directed between the interacting protons. In principle, the order parameter for the $\mathrm{C}-\mathrm{H}$ bond could be determined from measurements of ${ }^{13} \mathrm{C}-\mathrm{H}$ dipolar interactions, but no such measurements have been reported yet. 
In the case of a first order nuclear dipolar interaction between a pair of $I=\frac{1}{2}$ spins, the separation between the resonance lines, $\Delta v$, is given by (36)

$$
\begin{aligned}
\Delta v & =2 \Omega\left(\overline{\left.3 \cos ^{2} \theta-1\right)},\right. \text { where } \\
\Omega & =\frac{1}{2 \pi}\left(\frac{3 \gamma_{1} \gamma_{2} \hbar}{4 r^{3}}\right)
\end{aligned}
$$

$\gamma_{1}$ and $\gamma_{2}$ are the gyromagnetic ratios of the nuclei involved, and $\theta$ is the angle between the interaction vector $\mathbf{r}$ and the applied magnetic field. The average is over the range of angles the vector $\mathbf{r}$ traverses on the timescale of observation. When the motion allows the Hamiltonian to retain effective axial symmetry relative to a laboratory set of axes, then we may write

$$
\overline{3 \cos ^{2} \theta-1}=\frac{1}{2} \overline{\left(3 \cos ^{2} \beta-1\right)}\left(3 \cos ^{2} \theta^{\prime}-1\right) \text {. }
$$

Here $\theta^{\prime}$ is the angle between the applied magnetic field and the director, $\mathbf{d}$, and the angle, $\beta$, is between $\mathbf{r}$ and $\mathbf{d}$. The order parameter, $S_{\beta}$, is defined as

$$
S_{\beta}=\frac{1}{2} \overline{\left(3 \cos ^{2} \beta-1\right)} \text {. }
$$

In the case of an oriented sample, one then observes two resonance lines separated by

$$
\Delta v=2 \Omega S_{\beta} \overline{\left(3 \cos ^{2} \theta^{\prime}-1\right)} \text {. }
$$

For a nonoriented system where there is a uniform distribution of directors relative to the magnetic field, the splitting between the maxima in the composite NMR lineshape is given by

$$
\Delta v=2 \Omega S_{\beta} .
$$

Equation 1 is equally valid for the deuterium quadrupolar splitting in the case of an axially symmetric static quadrupolar interaction tensor except that

$$
\Omega=\frac{3 e^{2} q Q}{8 h},
$$

where $e^{2} q Q / h$ is the quadrupolar coupling constant. Note that $\mathbf{r}$ is now directed along the symmetry axis of the electric quadrupole interaction tensor.

In principle, order parameters for methylene segments or methyl tops in phospholipid systems can also be determined by measuring the chemical shift anisotropy of the ${ }^{13} \mathrm{C}$ nucleus. For phospholipid systems, the measured ${ }^{13} \mathrm{C}$ chemical shift anisotropies are quite small (27) and no detailed interpretations in terms of segmental order have been attempted. However, ${ }^{31} \mathrm{P}$ chemical shift anisotropies are larger, and these chemical shift anisotropy effects have, in fact, becn observed for the more ordered head group (28-34). The observed spectra are characteristic of a system with axial symmetry, even though the static shielding tensor for the ${ }^{31} \mathrm{P}$ nucleus is not axially symmetric. Because of the latter, two order parameters are required to completely describe the interaction, and, without further information, a quantitative interpretation of the observed "residual" ${ }^{1} \mathrm{P}$ chemical shift anisotropy is not possible. 
The importance of the coordinate transformation given by Equation 3 is that the relevant tensor interactions are reduced to a product of a scalar quantity, the order parameter, and a function describing the orientation of the director relative to the magnetic field. The order parameter reflects the motion of the vector $\mathbf{r}$ about the director and is independent of the orientation of the director. It is a measure of the distribution of the orientations of the vector $\mathbf{r}$ with respect to the director. Thus if $g(\beta)$ is the distribution function describing the orientation of $\mathbf{r}$ relative to the director,

$$
S_{\beta}=\frac{\frac{1}{2} \int_{0}^{\pi}\left(3 \cos ^{2} \beta-1\right) g(\beta) \sin \beta \mathrm{d} \beta}{\int_{0}^{\pi} g(\beta) \sin \beta \mathrm{d} \beta} .
$$

The exact form of the distribution function depends on the timescale of motion that is averaging the tensor interactions measured by the NMR experiment. Because of Equation 8, the value of $S_{\beta}$ depends on the interaction vector used to sample the motion. In principle, knowledge of the order parameter for a number of vectors could then be used to determine the distribution function.

The above discussion is only relevant for phospholipids in the liquid crystalline phase. The order parameter concept is not useful for the gel phase, where the motion is much more restricted and does not give rise to an effective axially symmetric Hamiltonian.

\section{Determination of Acyl Chain Order Parameters from ${ }^{2}$ H NMR Measurements. Acyl Chain Flexibility Gradients}

Order parameters have been determined from the deuterium quadrupolar splittings in selectively deuterated fatty acids and dipalmitoyllecithins (19-22, 24, 37). The order parameters determined for fatty acids intercalated into lecithin bilayers are nearly identical to those obtained for the deuterated phospholipids, which indicates that fatty acids can be used as reliable probes of membrane structure. The order parameters have been determined for different positions along the chain and these are shown in Figure 1.

The values of the order parameter determined from ${ }^{2} \mathrm{H}$ NMR experiments do not agree with those determined from ESR experiments $(1,2)$. The ESR measurements suggest a continuous flexibility gradient along the hydrocarbon chain, while ${ }^{2} \mathrm{H}$ NMR measurements indicate no gradient for the upper two thirds of the chain (Figure 1). These discrepancies have been attributed to perturbations on the system caused by the bulky nitroxide spin label used in the ESR experiments (19-22). However, as Gaffney \& McConnell (38) pointed out, the timescales of the NMR and ESR experiments are different, and the NMR experiment may sample additional motions. Consequently, there is no reason to expect the two sets of order parameters to have the same values.

The picture of the lipid bilayer drawn from spin label experiments $(1,2)$ is one in which each $\mathrm{C}-\mathrm{C}$ bond has an intrinsic flexibility due to rotational isomerizations 


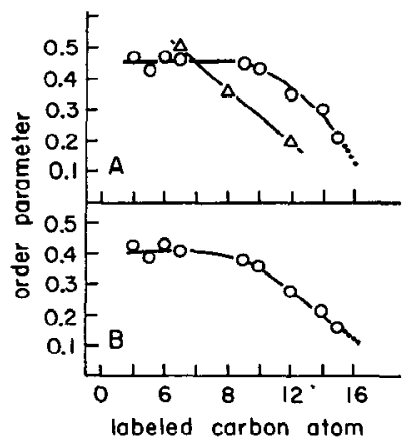

Figure 1 Order parameter $S_{\text {mol }} \sim 2 S_{\mathrm{CD}}$ of dipalmitoyllecithin (DPL) in multilayers as a function of the position of the labeled carbon atom. (A) $41^{\circ} \mathrm{C}:(\mathrm{O})$ deuterium data $(48.5 \mathrm{wt} \%$ DPL; $\left.51.5 \mathrm{wt} \% \mathrm{H}_{2} \mathrm{O}\right):(\triangle)$ ESR spin-label data $\left(20 \mathrm{wt} \%\right.$ DPL; $\left.80 \mathrm{wt} \% \mathrm{H}_{2} \mathrm{O}\right)$. (B) $50^{\circ} \mathrm{C}$ : (O) deuterium data $\left(48.5 \%\right.$ wt $\left.\% \mathrm{DPL} ; 51.5 \mathrm{wt} \% \mathrm{H}_{2} \mathrm{O}\right)$. Taken from Reference 20.

between trans $(t)$ and gauche $(g)$ conformations. One end of the chain is anchored in the lipid-water interface and is thus fixed, while the other end is free to move. The overall motion is then determined by the superposition of segmental motions. Near the polar surface the motion is quite restricted, while towards the center of the bilayer the motion is increasingly more random because of the increasing number of flexible $\mathrm{CH}_{2}$ units.

The deuterium order parameters suggest a somewhat different picture of the bilayer, requiring conformational changes which leave the acyl chains essentially parallel. One such conformation, called a kink $(39,40)$, arises as a result of $\beta$-coupled trans-gauche rotations $\left(g^{+} \mathrm{tg}^{-}\right.$or $g^{-} \mathrm{tg}^{+}$sequences). When a kink is formed, part of the lipid chain is displaced perpendicular to the molecular axis. The trans segments on either side of the kink remain parallel, but are displaced from their positions in the all-trans chain by approximately $1.5 \AA$. At the same time, the chain is shortened by the length of one $\mathrm{CH}_{2}$ unit. The suggestion that kink formation is energetically more favorable than isolated trans-gauche isomerizations is based on the argument that this structure minimizes unfavorable interactions between neighboring chains. On the basis of deuterium order parameters Seelig \& Seelig (20) have estimated that the probability, $p_{t}$, of a carbon-carbon bond being in a trans conformation is approximately 0.7 . This means that, on the average, a palmitic acid acyl chain contains four gauche bonds or two kinks.

The order parameters measured by ${ }^{2} \mathrm{H}$ NMR also serve as a convenient starting point for testing theoretical models for lipid bilayers. Using the mean molecular field model, Marčelja (41) has successfully calculated deuterium order parameters for soap-like bilayers. Schindler \& Seelig (42) have applied Marčelja's model to phospholipid bilayers and satisfactorily reproduced the deuterium order parameters measured for these systems. The Marčelja model predicts a $p_{t}$ of 0.69 , which is in agreement with Seelig \& Seelig's (20) earlier estimates for phospholipid systems, and also in agreement with Marčelja's (41) estimates of $p_{t}$ in soap-like systems. 
The Marçelja model predicts an average of $0.6 \mathrm{kinks}$ and $0.2 \mathrm{jogs}\left(g^{+} \mathrm{ttg}-\right.$ sequences) per phospholipid chain, plus a number of additional conformations of lesser statistical importance (20).

All of the above interpretations of phospholipid bilayer order parameters assume that intramolecular motions provide the only significant mechanism for the reduction in order. No consideration is given to rigid-body type motions of the chain, and, in the past, the tendency has been to dismiss the importance of these types of motion in lipid bilayers (20). However, studies of liquid crystal systems by Ukleja et al (43) indicate that amphiphilic molecules can reorient about an average director. If similar reorientations of the lipid molecules, or the chains of lipid molecules, take place in the bilayer, the effective symmetry axis must be identified with the average chain orientation rather than with the instantaneous orientation.

Recently, Petersen \& Chan (44) proposed a model for phospholipid motion in which both chain isomerization and chain reorientation are important mechanisms in the reduction of molecular order. With the assumption that reorientation of the methylene group is axially symmetric with respect to the instantaneous chain axis, and that the timescales of chain isomerization and chain reorientation are very different, the order parameter could be rewritten as

$$
S_{\beta}=\frac{1}{2}\left(3 \cos ^{2} \beta-1\right)=\left[\frac{1}{2}\left(3 \cos ^{2} \alpha-1\right)\right] \cdot\left[\frac{1}{2}\left(3 \cos ^{2} \gamma-1\right)\right]=S_{\alpha} S_{\gamma},
$$

where the angles are defined as in Figure 2. An important consequence of Equation 9 is that the order parameter predicted from chain isomerization calculations will be scaled by a factor $S_{\alpha}$, which will be approximately constant for the whole chain. Thus, the relative order parameter for various segments along the chain could probably be predicted quite well by models based only on chain isomerization $(41,42,45)$. The key difference between the Petersen-Chan model (44) and those of

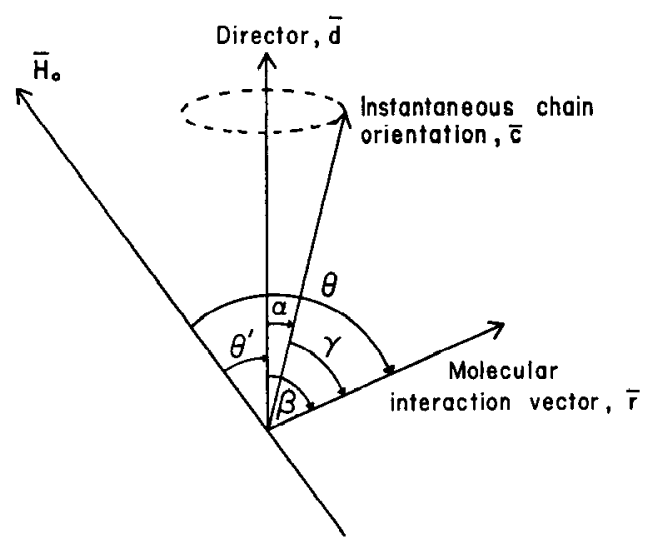

Figure 2 Illustration of the vectors and angles relevant to the lipid chain motional model. $\mathbf{H}_{\mathbf{0}}$ is the direction of the applied magnetic field. The director, $\mathbf{d}$, is normal to the bilayer surface. Taken from Reference 44. 


\section{BOCIAN \& CHAN}

Schindler \& Seelig (42) and Marčelja (41) is the manner in which the order parameter is scaled. The latter workers are forced to invoke a rapid equilibrium among a distribution of orientations of the first methylene segments in order to fit their calculations to the observed order parameters. As a result, the probabilities obtained for the various orientations of a methylene segment relative to the bilayer normal depend upon the orientation of the first segment, and the probability that a segment is in the all-trans conformation depends on the distribution chosen for the orientation of the first segment. While Schindler \& Seelig assume that the equilibrium between the orientations of the first segment is fast on the NMR timescale, they make no distinction between the rate of isomerization of the first segment and the rate of isomerization along the chain. It is possible that rearrangement of the first segment, which may correspond to chain reorientation in the Petersen-Chan model, occurs on a much different timescale than chain isomerization-slow on the ESR timescale, for instance. Also, since the chain reorientations are probably cooperative, involving a large number of lipid molecules, the probability of having a trans segment should be independent of the chain orientation. In all probability $S_{\alpha}$ differs between $S_{\mathrm{CD}}$ and $S_{\text {ESR }}$. This is likely becausc the timescale of an NMR experiment is much longer than that of an ESR experiment. In contrast, the timescales of ${ }^{1} \mathrm{H}$ and ${ }^{2} \mathrm{H}$ NMR measurements are comparable, and the contribution to the respective order parameters from $S_{\alpha}$ should be the same.

\section{Order Parameters Estimated from Proton NMR. Analysis of Free Induction Decays and NMR Lineshapes}

The first ${ }^{1} \mathrm{H}$ NMR spectra of aqueous dispersions of amphiphilic molecules were obtained by Lawson \& Flautt $(46,47)$. The spectrum is characterized by a sharp central peak and extremely broad wings. The wings were, in fact, so pronounced that Lawson \& Flautt called the band shape "super-Lorentzian" (46, 47). Lawson \& Flautt originally proposed that the band shape arose from the superposition of proton resonances from different methylene groups along the chain, with the individual resonance lines becoming increasingly narrow from the head to the tail of the chain because of increased motional averaging of the dipolar interactions. Kaufman et al (48) concluded that the spectral broadening arose because of internal magnetic field gradients, while Hansen \& Lawson (49) concluded that diffusion through the local magnetic field inhomogeneities was the source of the band shape. Chan et al (50) showed that these interpretations were incorrect and that the origin of the observed band shape is the incomplete averaging of static dipolar interactions present in the anisotropic phase.

A number of attempts have been made to determine theoretical expressions for the complex lineshapes observed for amphiphilic molecules in the liquid crystalline state $(14,18,51-55)$. All of these treatments argue that, in addition to incomplete averaging of static dipolar interactions, effective axial symmetry about the bilayer normal is necessary for the observation of the super-Lorentzian band shape. Charvolin \& Rigny (51) have simulated the free induction decay (FID) observed for aqueous soap systems by a superposition of two Gaussian and one Lorentzian 
absorption curve. They assumed that the Gaussian curves originated from dipolar couplings not averaged to zero, while the Lorentzian curve originated in dynamic processes. Wennerström (53) has quantitatively reproduced the super-Lorentzian curve observed in aqueous soap systems by simply integrating an expression of the form

$$
L\left(v-v_{0}\right)=\int_{0}^{1}\left|3 \cos ^{2} \theta^{\prime}-1\right|^{-1} f\left[\left(v-v_{0}\right) /\left|3 \cos ^{2} \theta^{\prime}-1\right|\right] d \cos \theta^{\prime},
$$

where $f\left[\left(v-v_{0}\right) /\left|3 \cos ^{2} \theta^{\prime}-1\right|\right]$ is Gaussian. Ulmius et al (14) have extended the method of Wennerström to phospholipid systems by assuming a superposition of three independent Gaussian components, which represent the methylene and glycerol backbone, terminal methyl, and choline methyl protons, respectively. Bloom and co-workers $(54,55)$ also have developed expressions for describing the FID in lyotropic systems using the method of moments (36). They showed that the FID for a randomly oriented set of domains with axial symmetry can be expressed as follows

$$
\begin{aligned}
S_{1}(t) & =S_{1}(0) \int_{0}^{\pi / 2} G_{\theta^{\prime}}(t) \sin \theta^{\prime} \mathrm{d} \theta^{\prime}, \text { where } \\
G_{\theta^{\prime}}(t) & =\sum_{n=0}^{\infty} \frac{(-1)^{n}}{(2 n) !} M_{2 n}\left(\theta^{\prime}\right) t^{2 n} .
\end{aligned}
$$

In Equation 12, the observed moments $M_{2 n}\left(\theta^{\prime}\right)=M_{2 n}(0)\left[P_{2}\left(\cos \theta^{\prime}\right)\right]^{2 n}$ are the residual moments of the absorption line whose shape is determined from the timeaveraged dipolar Hamiltonian. In their analysis, Bloom and co-workers (55) compared the experimental FID with that calculated by assuming three different forms for the second moment of the absorption line: 1. a simple Gaussian, 2. a superposition of Gaussians centered near zero frequency in the rotating frame, and 3. a superposition of Gaussian doublets arising from intrapair dipolar interactions, symmetrically displaced from zero frequency. Both the Gaussian and superposition of Gaussian doublets resulted in reasonable agreement with experiment, while the superposition of Gaussians did not. By extending their analysis to the calculation of the shape of Jeener echoes (56), Bloom et al (55) showed that a superposition of Gaussian doublets gave results more in accord with the observed FID than did the simple Gaussian.

All of the above attempts at calculating the lineshape in lyotropic systems suffer from the fact that the lineshape functions are unknown, and there is no reason a priori to assume that this shape is Gaussian. In addition, most of the treatments offer little insight into the relationship between the resonance lineshape (or the FID) and the order parameter. While it is true that proton order parameters could be measured directly with selectively protonated perdeuterated phospholipids, this method also has its difficulties since the phase transition temperature is shifted when protons are replaced by deuterons (57). Attempts have been made to estimate the order parameter of natural membranes by simulating the early part of the FID, taking into account both inter- and intrapair dipolar interactions. In the Seiter-Chan treatment (18), the stochastic linewidth theory of Anderson (58) was modified to 


\section{BOCIAN \& CHAN}

include restricted anisotropic motion. The geminal dipolar interactions were treated exactly, while interpair interactions were treated in an approximate way. Thus, the observed spectrum is a superposition of broadened Pake doublets (36), modified by the interactions between the different pairs. By computing the early part of the FID, Seiter \& Chan obtained estimates of the order parameter for the methylene protons in the upper part of the hydrocarbon chain.

More recently, Hagan et al (P. S. Hagan, N. O. Petersen, S. I. Chan, unpublished results) simulated the FID for the upper part of the hydrocarbon chain, where the order parameter is nearly constant, by treating exactly the dipolar interactions between five geminal pairs of protons. The corresponding spectra show a modified Pake doublet band shape, but with contributions at zero frequency (in the rotating frame). These results are similar to those obtained by Seiter \& Chan (18), except that the central component is much less pronounced in the latter treatment. The difference between the results of Hagan et al and Seiter \& Chan is probably the result of an underestimation of the interpair interactions by the latter.

The results of Hagan et al also show that the early part of the FID is primarily sensitive to the wings of the broadened Pake doublet, while the central component contributes to the FID at longer times. Bloom and co-workers (55) showed that the FID exhibits a $t^{-1}$ dependence at long times. The results of Hagan et al confirm this dependence, but the calculated magnitude of the $t^{-1}$ component is smaller than is experimentally observed. This indicates that a sizable contribution to the experimentally observed intensity in the "central spike" of the super-Lorentzian band shape results from contributions by proton pairs with considerably smaller order parameters.

Independent of their calculations for the methylene protons, Seiter \& Chan (18) simulated the band shape for the methyl rotors in phospholipid systems. In the past, the tendency has been to overlook the importance of these groups (choline and acyl chain methyls) in determining the heterogeneous band shape, although these protons contribute $20-30 \%$ of the total observed spectral intensity. The simulated spectra for the methyl protons consists of a narrow resonance superimposed on a much broader resonance, with the narrow resonance accounting for $\sim 50 \%$ of the methyl intensity and contributing to the FID at long times.

\section{Interpretation of the Order Parameter}

If there is more than one type of motion contributing to the reduction in order, the measurement of the order parameter for a single interaction vector does not allow a complete description of the motion of the system. This is probably the case for phospholipid systems, where rigid body motions of the chain, as well as trans-gauche isomerization, may be important. However, if two different types of interaction vectors are measured, a more complete description of the order can be obtained, provided the interaction vectors sample the same distribution of orientations. Following the notation of Petersen \& Chan (44), we use $g(\gamma)$ and $g(\alpha)$ to denote the distribution functions for chain isomerization and chain reorientation, respectively. 
The distribution function for chain isomerization can be expressed in terms of discrete trans and gauche conformers $(1,20)$.

$$
g(\gamma)=p\left(\gamma_{\mathrm{i}}\right) \delta\left(\gamma-\gamma_{\mathrm{i}}\right) \text {, with } \Sigma p\left(\gamma_{\mathrm{i}}\right)=p_{t}+2 p_{g}=1,
$$

where $p\left(\gamma_{i}\right)$ is the population fraction for a given conformation and $\gamma_{i}$ is the angle between the interaction vector, $\mathbf{r}$, and the instantaneous chain orientation (Figure 2). Depending on the vector being sampled, the order parameters for chain isomerization, $\boldsymbol{S}_{\gamma}$, are different, and these can be expressed as follows:

$$
\begin{aligned}
S_{\gamma}^{\mathrm{HH}}= & \frac{3}{8} p_{t}-\frac{1}{8} \text { for nuclear dipolar interaction; } \\
S_{\gamma}^{\mathrm{CD}}, S_{\gamma}^{\mathrm{CH}}= & -\frac{1}{2} p_{t} \text { for symmetric deuterium quadrupolar } \\
& \text { and }{ }^{13} \mathrm{C}-{ }^{1} \mathrm{H} \text { dipolar interaction; } \\
S_{\gamma}^{\mathrm{ESR}}= & { }_{8}^{9} p_{t}-\frac{1}{8} \text { for the acyl-chain nitroxide radical. }
\end{aligned}
$$

Although the values of $S_{\gamma}$ vary with the interaction vector used to sample the motion, the contribution to the chain reorientation order parameter, $S_{\alpha}$, should be the same provided that the different measurements sample the same distribution of instantaneous chain orientations. On this basis, Petersen \& Chan (44) have compared the proton and deuterium order parameters for the upper part of the acyl chains in phospholipids. The experimental values for $S_{\mathrm{CD}}$ for the first ten segments of the hydrocarbon chain are quite similar and have an average value of about $-0.20 \pm$ $0.01(20,24)$. The $S_{\mathrm{HH}}$ inferred from the early part of the FID in ${ }^{1} \mathrm{H}$ spectra is about $-0.17 \pm 0.04$ (18). This implies $p_{t}$ is close to unity, in contrast to the value of 0.7 obtained by considering only chain isomerizations as an effective mechanism for the reduction in order $(20,41,42)$. More specifically, they concluded

$$
1 \geq p_{t} \geq 0.8
$$

with

$$
-0.5 \leq \mathrm{S}_{\gamma}^{\mathrm{CD}} \leq-0.40
$$

or

$$
-0.5 \leq S_{y}^{\text {HH }} \leq-0.43
$$

and

$$
0.38 \leq S_{\alpha} \leq 0.53 \text {. }
$$

Consequently, Petersen \& Chan concluded that chain reorientation must make a substantial contribution to the reduction in order in phospholipid bilayer systems. Using distribution functions of the form

$$
g(\alpha)=\text { constant for } 0 \leq \alpha \leq \Delta \alpha
$$

and

$$
g(\alpha)=\text { constant } \mathrm{e}^{-1 / 2\left(\alpha / \alpha_{0}\right)^{2}},
$$

where $\Delta \alpha$ is the maximum value of $\alpha$ for the rectangular distribution function and $\alpha_{0}$ is the most probable value of $\alpha$ for the Gaussian distribution, the range of values 
for $S_{\alpha}$ corresponded to ranges of $50^{\circ} \leq \Delta \alpha \leq 60^{\circ}$ and $27^{\circ} \leq \alpha_{0} \leq 34^{\circ}$ for the two distribution functions, respectively.

Niederberger \& Seelig (59) have measured deuterium order parameters for the lyotropic liquid crystalline phase of long chain fatty acids and found that they are about $50 \%$ larger than those of the lipid bilayer, which suggests a more ordered phase in the fatty acid system. Seelig \& Niederberger (22) estimated a value for $S_{\alpha}$ of about $0.7-0.8$, which corresponds to a $\Delta \alpha$ of $35^{\circ}$. The difference between the two systems then is a smaller angular excursion of the chain in the case of the fatty acids, presumably because of the more regular packing of the acyl chains in their liquid crystalline phase.

An important consequence of the Petersen-Chan model (44) is that the most probable orientation of the acyl chain is not parallel to the bilayer normal $\left(\alpha_{0}>0\right)$. As a consequence, the ESR spectra, which probably sample a static distribution of chain orientations, could be interpreted on the basis of permanently tilted chains $(38,60)$. However, as Petersen \& Chan emphasized, the same spectral effects could arise from a statistical distribution of chains when the distribution is sampled on a short timescale. Also, there is no way to ascertain from the NMR results whether there is simply a distribution of chains about the bilayer normal, or a distribution about a permanently tilted orientation, provided the chains rotate about the director in the latter case.

The inclusion of chain reorientation in modeling phospholipid motions in bilayers is also in accord with recent Raman and fluorescence investigations (61-63). The Raman studies $(61,62)$ are compatible with large values of $p_{t}$ in the liquid crystalline phase. These studies also indicate that there are large changes in intermolecular order upon passing through the gel-liquid crystalline phase transition. Recent fluorescence measurements (63) indicate that inclusion of an off axis wobble of the chain is necessary to successfully interpret these experimental data.

\section{Sonicated Lipid Bilayer Vesicles-Curvature Effects}

When aqueous dispersions of phospholipids are sonicated, the effect is to produce sealed single-walled bilayer vesicles (radius $\approx 125-150 \AA$ ) whose NMR spectra are characterized by high resolution features (3-11). Thus, for these systems, nuclear dipolar and quadrupolar interactions and chemical shift anisotropies are averaged to zero on a timescale of at least $10^{-5}$ seconds. Because of the high resolution features of their NMR spectra, bilayer vesicles have been utilized extensively as membrane models in magnetic resonance studies.

The quantitative interpretation of the NMR spectra of sonicated bilayers has led to some notable controversies in the literature, centering around the origin of the narrowing of the resonance lines. Finer and co-workers (64-66), Bloom et al (54), and more recently Wennerström \& Ulmius (67), and Stockton et al (24) have interpreted the linewidth reduction as an effect of the rapid overall isotropic tumbling of the vesicles. Bloom et al (54), and Wennerström \& Ulmius (67) have, in fact, derived theoretical expressions for vesicle lineshapes. On the other hand, Chan and co-workers $(17,18,44,68,69)$, and Horowitz et al $(70)$ have argued that overall 
tumbling is too slow to produce the narrow resonances observed in vesicle spectra, and that the narrow lines occur as a result of additional disruption in the bilayer order caused by the extreme surface curvature in the vesicles. Finer's conclusions (66) were reached on the basis of a treatment of vesicle lineshapes that used the method of moments introduced by Gutowsky \& Pake (71), and modified to meet the present situation. Chan and co-workers' (18) opposite conclusions were based on a treatment that used the stochastic linewidth theory of Anderson (58), modified to account for both the restricted motion of the fatty acid chains and the Brownian tumbling of the bilayer. Later Chan and co-workers (69) reviewed both Finer's and their own treatment, and demonstrated that the Gutowsky-Pake method was inappropriate for treating lipid bilayer systems, and that Finer's treatment also was not consistent with the experimental data. Petersen \& Chan (44) noted that, although the treatments of both Bloom et al (54), and Wennerström \& Ulmius (67) adequately account for the modulation by vesicle tumbling of the heterogeneous contribution to the linewidth arising from residual static dipolar interactions, they fail to take proper account of the effects of the homogeneous contribution to the linewidth, which is present because of extensive chain motions, even in the absence of vesicle tumbling.

There have been a number of attempts to quantify the importance of vesicle tumbling on the NMR linewidths by examining the effects of viscosity changes on the spectra $(28,69,70)$. However, no viscosity dependence has been found for ${ }^{1} \mathrm{H}$ NMR linewidths. Stockton et al (24) have suggested that the lack of viscosity dependence indicates that the rate of vesicle tumbling is controlled by the microviscosity of the vesicles, rather than by the bulk solution viscosity. Recently, however, Cullis (72) has observed a marked viscosity dependence of the ${ }^{31} \mathrm{P}$ NMR linewidths of the glycerol phosphate moiety in sonicated bilayer vesicles. Thus, the mechanisms contributing to ${ }^{31} \mathrm{P}$ linewidth are at least in part modulated by the overall tumbling of the bilayer unit. This clearly indicates that arguments based on microviscosity are unfounded. In addition, Cullis (72) has determined the lateral diffusion coefficient for lipids in bilayer vesicles to be $D \approx 2 \times 10^{-8} \mathrm{~cm}^{2} / \mathrm{sec}$, which is approximately the same as that determined for multilamellar arrays (73). This result also implies that lateral diffusion cannot contribute significantly to the observed line narrowing (see below).

More recently, Curatolo et al (74) have obtained ${ }^{13} \mathrm{C}$ spectra of glycolipid vesicles. These spectra show narrow lines characteristic of the spectra of other phospholipid vesicles. When lectin is added to the solution, the glycolipid vesicles are agglutinated, and electron microscopy shows that the vesicles are completely immobilized. However, the spectra of the immobilized vesicles still contain narrow lines, indicating that some mechanism other than vesicle tumbling must be responsible for the line narrowing. In addition, Lichtenberg \& Zilberman (D. Lichtenberg, Y. Zilberman, private communication) have obtained ${ }^{1} \mathrm{H}$ NMR spectra of mixed micelles of lecithin and deoxycholate. These micelles exist as flat bilayers of lecithin surrounded by cholate. For flat bilayers whose radius is of the same order as sonicated vesicles (125 A), Lichtenberg \& Zilberman observe broad resonances in the NMR spectrum. The ${ }^{1} \mathrm{H}$ linewidths in the micelles only approach those observed for sonicated 
vesicles $(20-30 \mathrm{~Hz})$ when the micelle radius is extremely small $(29 \AA)$, where the correlation time for micelle tumbling is at least 50 times faster than that of a sonicated bilayer vesicle.

Other recent spectroscopic investigations also support the view that the high surface curvature of the vesicles results in additional disorder in the bilayer. Raman studies $(61,62)$ indicate that, although above the phase transition there is no gross difference in the intramolecular order between multilamellar and vesicular dispersions, there exist large differences in intermolecular order.

Petersen \& Chan (44) have recently evaluated the effects of surface curvature in the context of their model for lipid motion, which includes both chain isomerization and chain reorientation. On this basis, they reiterate the original conclusions of Chan and co-workers $(17,18,68,69)$ that the high surface curvature in the vesicular systems must result in additional disorder within the bilayer. In terms of the PetersenChan model (44), Hagan et al (P. S. Hagan, N. O. Petersen, S. I. Chan, unpublished results) have shown that the linewidth for an isolated methylene segment that is undergoing restricted anisotropic motion is given by

$$
\begin{aligned}
\Delta v_{H}= & \frac{1}{\pi T_{2}}=\frac{4}{5 \pi}\left(\frac{3 \gamma^{2} \hbar}{4 r^{3}}\right)\left(S_{\gamma}^{\mathrm{HH}}\right)^{2}\left[S_{\alpha}^{2} \tau_{e}+\frac{1}{9}\left(\frac{5}{2} \sigma_{\alpha}^{2}-5 S_{\alpha}^{2}-2 S_{\alpha}\right.\right. \\
& \left.\left.+9 \mu_{\alpha}^{2}+7\right) \tau_{\perp}\right],
\end{aligned}
$$

where $S_{\gamma}^{\mathrm{HH}}$ and $S_{\alpha}^{\mathrm{HH}}$ are the order parameters defined previously, $\mu_{\alpha}$ is the mean of the function $\sin 2 \alpha(t)$, and $\sigma_{\alpha}^{2}$ is the variance of the function associated with the molecular axis reorientation, namely $\sigma_{\alpha}^{2}=\overline{\left(\frac{3}{2} \cos ^{2} \alpha(t)-\frac{1}{2}\right)^{2}}-S_{\alpha}^{2}$. The correlation time $\tau_{\perp}$ is associated with the rate of chain reorientation and is assumed to be much slower than the correlation time, $\tau_{\|}$, associated with chain isomerizations, which occur via kink diffusion in the Petersen-Chan model. The correlation time, $\tau_{e}$, is the effective correlation time associated with those motions modulating the residual static dipolar interactions associated with the otherwise stationary bilayer unit; more specifically $\left(1 / \tau_{c}\right)=:\left(1 / \tau_{v}\right)+\left(1 / \tau_{d}\right)$, where $\tau_{v}$ is the rate of vesicle tumbling and $\tau_{d}$ is the rate of lateral diffusion of the lipid molecules on the spherical vesicle surface. It is also assumed in the derivation of Equation 18 that the correlation time, $\tau_{\perp}$, associated with the chain reorientation relative to the director is at least an order of magnitude faster than $\tau_{e}$. A third contribution to the linewidth arises from spinlattice relaxation, but, since $\left(1 / T_{2}\right) \gg\left(1 / T_{1}\right)$ for anisotropic motion, it can be ignored. Note that in the absence of chain reorientation relative to the director, the PetersenChan linewidth expression reduces to

$$
\Delta v_{\mathrm{H}}=\frac{4}{5 \pi}\left(\frac{3 \gamma^{2} \hbar}{4 r^{3}}\right)^{2} S_{\mathrm{HH}}^{2} \tau_{e}
$$

which is identical to expressions commonly used to calculate linewidths in sonicated vesicles (54).

The derivation resulting in Equation 18 is strictly valid only for isolated pairs of protons and does not account for interpair dipolar interactions. For a hydro- 
carbon chain where there is a distribution of order parameters along the chain and dipolar interactions among the various geminal pairs, the composite methylene signal is actually a superposition of Lorentzians rather than a single Lorentzian resonance. Note that this is true even in the case of weakly interacting geminal pairs. Wennerström \& Ulmius (67) have treated the many-body problem in a formal way and have shown that the resultant band shape for the small vesicle is given by the following general expression

$$
F(\omega)=\int_{-\infty}^{\infty} T_{2, \text { eff }}\left(\omega^{\prime}\right) I\left(\omega^{\prime}\right) /\left[1+\left(\omega-\omega_{0}\right)^{2} T_{2, \text { eff }}\left(\omega^{\prime}\right)\right] \mathrm{d} \omega^{\prime},
$$

where $I\left(\omega^{\prime}\right)$ is the spectrum of the hydrocarbon chain when its director is oriented at $0^{\circ}$ with respect to the applied magnetic field. For each transition $\alpha \rightarrow \alpha^{\prime}$ that contributes to the band shape $I\left(\omega^{\prime}\right)$ at frequency $\omega^{\prime}$, there is a contribution to the resultant vesicle spectrum of a Lorentzian with width

$$
\begin{aligned}
1 / T_{2, \text { erf }}\left(\omega^{\prime}\right) & =1 / T_{2, \text { eff }}^{\alpha \alpha^{\prime}} \\
& =\left(\omega_{\alpha}^{D}-\omega_{\alpha^{\prime}}^{D}\right) \tau_{v} / 5+1 / T_{2}^{o},
\end{aligned}
$$

where $\omega_{\alpha}^{D}$ is the eigenvalue of the many-body dipolar Hamiltonian in the $\alpha$ eigenstate and $1 / T_{2}^{0}$ is related to the homogeneous width in the otherwise stationary bilayer unit. As expected, the calculated lineshape for the vesicle is very sensitive to the details of $I\left(\omega^{\prime}\right)$, with the latter being rather more sensitive to the extent of overall molecular order than to the distribution of segmental order along the chain, or to the strength of the interpair dipolar interactions. For this latter reason, it should then be possible to obtain a reasonable estimate of the width of the vesicle spectrum using Equation 18, particularly when close to $100 \%$ of the expected intensity is observed within a spectral width several times the apparent width. Moreover, $I\left(\omega^{\prime}\right)$ is in practice unknown for the hydrocarbon chain, either in the unsonicated sample or in the bilayer vesicle, and it is questionable whether it is transferable from one model membrane to the other because of intrinsic curvature effects in the sonicated bilayer vesicle.

In any case, the Petersen-Chan theory is essentially exact for the deuterium linewidth observed in ${ }^{2} \mathrm{H}$ experiments, for which

$$
\Delta v_{D}=\frac{9 \pi}{20}\left(\frac{e^{2} q Q}{h}\right)\left(S_{\gamma}^{\mathrm{CD}}\right)^{2}\left[S_{\alpha}^{2} \tau_{e}+\frac{1}{9}\left(\frac{5}{2} \sigma_{\alpha}^{2}-5 S_{\alpha}^{2}-2 S_{\alpha}-9 \mu_{\alpha}^{2}+7\right) \tau_{\perp}\right] .
$$

This expression reduces to that used by Stockton et al (24)

$$
\Delta v_{D}=\frac{9 \pi}{20}\left(\frac{e^{2} q Q}{h}\right) S_{\mathrm{CD}}^{2} \tau_{v}+\frac{1}{\pi T_{1}}
$$

in the limit that chain reorientation and lateral diffusion are not important motions. Using the values of $S_{\gamma}^{\mathrm{CD}}, S_{\alpha}$, and $\tau_{\perp}$ estimated from the multilayer data and the expected value of $\tau_{e}$, one calculates a $\Delta v_{D}$ for the methylene segments at the upper part of the chain that is about a factor of 5 too large compared with the width of $550 \pm 50 \mathrm{~Hz}$ observed (24). Stockton et al (24) have also correlated the deuterium linewidths of selectively deuterated fatty acids incorporated into vesicles, and the 
order parameters in multilayers for the same lipid system as a function of position of the deuterium along the chain. They found that the vesicle linewidths for different positions vary linearly with the square of the multilayer order parameter measured for the same position in the chain, and argued on this basis that there is no reduction in the order parameter upon sonication. The Petersen-Chan model predicts this correlation if 1 . there is no change in the intramolecular order parameter, $S_{\gamma}^{\mathrm{CD}}$, upon sonication, or 2 . if the flexibility gradient changes proportionally along the chain. The correlation observed by Stockton et al (24) is independent of the values of $S_{\alpha}$, or changes in it, since $S_{\alpha}$ applies to the whole chain.

From Equations 18 and 22, one predicts for the proton and deuterium linewidths a ratio of

$$
\left(\Delta v_{\mathrm{H}} / \Delta v_{\mathrm{D}}\right)=0.016\left(S_{\gamma}^{\mathrm{HH}} / S_{\gamma}^{\mathrm{CD}}\right)^{2} \cong 0.016
$$

since $S_{\gamma}^{\mathrm{HH}} \approx S_{\gamma}^{\mathrm{CD}}(44)$. This result is in reasonable agreement with the observed ratio of 0.036 , considering that the proton linewidth is for the entire acyl chain and the dispersion in chemical shifts as well as spin-spin couplings will make the resonances appear broader, and would seem to indicate that proton NMR linewidths of small vesicles can be predicted quite well with Equation 18.

As far as the ${ }^{1} \mathrm{H}$ NMR spectra of vesicles are concerned, one typically observes a methylene linewidth of $20-30 \mathrm{~Hz}$ with nearly $100 \%$ of the intensity observed. This is also true for the signal due to the $\alpha \mathrm{CH}_{2}$ 's adjacent to the carbonyls of the fatty acid chains. In addition, both signals exhibit no viscosity dependence. Petersen \& Chan (44) argue that both of these observations can only be satisfied if $\Delta \alpha \geq 70^{\circ}$ and $\tau_{\perp} / \tau_{v}$ is small. This represents an increase in $\Delta \alpha$ by at least $10-20^{\circ}$ in going from multilayers to small bilayer vesicles and a subsequent reduction in the order parameter by a factor of $2-3$ to -0.07 to -0.1 . Concomitant with the reduction in order, the correlation time for chain reorientation must also decrease from $10^{-7}$ to $10^{-8}$ $10^{-9}$ seconds.

The effects of the severe surface curvature in small bilayer vesicles are also directly reflected by the structural and dynamical differences measured for lipids in the inner and outer monolayers of small vesicles. The two halves of the bilayer have radii of curvature different in both sign and magnitude. Both the chemical shifts $(28,75)$ and the spin-lattice relaxation rates (76) of the choline methyl protons are different for these two regions. The differences in packing of the hydrocarbon chains in the two halves of the bilayer have also been shown, by the observation of different ${ }^{19} \mathrm{~F}$ chemical shifts for selectively fluorinated lipids intercalated into vesicles (25). On the basis of ${ }^{31} \mathrm{P}$ NMR and other measurements, Chrzeszczyk et al (77) have proposed that the curvature in small vesicles results in $25 \%$ reduction of the effective thickness of the inner monolayer relative to the outer monolayer. These workers suggest that the differential thickness of the monolayers is due to additional kinks in the inner monolayer, but such differences could also arise from a difference in the distribution of chain orientations in the two regions. All of the above observations further illustrate that the packing of phospholipid molecules in small vesicles is intrinsically different from that in multilamellar structures. 


\section{DYNAMICS}

\section{Acyl Chain Dynamics}

Measurement of the order parameter provides a means of determining the degree of motional restriction in the bilayer system, but the motional state of the system is not completely specified until the timescales of the molecular motions are determined. This information can be inferred from spin-lattice and spin-spin relaxation time measurements.

Spin-lattice relaxation times, $T_{1}$, provide information on molecular motions which occur on the timescale of the inverse Larmor frequency or less. Since the homogeneous spin-spin relaxation time, $T_{2}$, depends on the spectral density at zero frequency instead of at the Larmor frequency, it is sensitive to the slower motions in anisotropic systems, particularly those motions with correlation times longer than the reciprocal Larmor frequency. Under these circumstances, the homogeneous $T_{2} \ll T_{1}$ and slow motions usually have only a small effect on $T_{1}$. It is for this reason that one does not observe a dramatic change in the spin-lattice relaxation time in passing through the gel-liquid crystalline phase transition $(6,8,15,17,78,79)$.

Proton NMR $T_{1}$ measurements $(6,8,15,17)$ have shown that the FID of the chain methylene protons in multilayers is approximately a single exponential, suggesting that spin diffusion may be an important relaxation mechanism in these systems. In phospholipids the terminal methyl and the adjacent three or four methylene groups would probably act as the heat sink (17). Although the importance of spin diffusion as an ${ }^{1} \mathrm{H}$ relaxation mechanism has not been fully established, spin diffusion is definitely not an important mechanism in determining ${ }^{13} \mathrm{C}$ relaxation times, since each ${ }^{13} \mathrm{C}$ nucleus is generally bonded to a ${ }^{12} \mathrm{C}$, which disrupts the diffusion process.

Cabon-13 spin-lattice relaxation times have been measured for both multilayers and small bilayer vesicles, and are similar in the two systems (79-83). In both multilayers and vesicles, the $T_{1}$ 's increase with increasing temperature, which is analogous to the results obtained for these systems with ${ }^{1} \mathrm{H}$ NMR $(4,6,76,84)$. In addition, ${ }^{1} \mathrm{H}$ spin-lattice relaxation times for multilayer dispersions, and ${ }^{1} \mathrm{H}$ and ${ }^{13} \mathrm{C}$ spin-lattice relaxation times for vesicles increase with increasing frequency of irradiation $(4,6,76,81,84)$. All of the above measurements indicate that the chain motions which dominate $T_{1}$ are similar in multilayer dispersions and small bilayer vesicles. The ${ }^{13} \mathrm{C}$ spin-lattice relaxation times measured for individual carbons in small bilayer vesicles are shown in Table 1 . The relaxation times increase steadily in going from the upper part of the acyl chain to the terminal methyl group, which is consistent with the notion of increased mobility toward the center of the bilayer. Similar $T_{1}$ gradients are observed for ${ }^{2} \mathrm{H}$ relaxation in selectively deuterated fatty acids intercalated into phospholipid bilayers (24).

In order to account for the observed temperature and frequency dependence of the spin-lattice relaxation times, the dipolar interaction responsible for relaxation must be modulated by at least two motions with correlation times, $\tau_{\|}$and $\tau_{\perp}$, such that $\left(\omega_{0} \tau_{\|}\right)^{2} \ll 1$ and $\left(\omega_{0} \tau_{\perp}\right)^{2} \gg 1$. Under these conditions Kroon et al (76) have 
Table 1 Carbon-13 spin-lattice relaxation times for lecithin in sonicated vesicles ${ }^{a}$

\begin{tabular}{ll}
\hline \multicolumn{1}{c}{ Segment } & $T_{1}$ (sec) \\
\hline$-\mathrm{CH}_{3}$ & 2.8 \\
$-\mathrm{CH}_{2} \mathrm{CH}_{3}$ & 1.4 \\
$-\mathrm{CH}_{2} \mathrm{CH}_{2} \mathrm{CH}_{3}$ & 0.64 \\
$-\left(\mathrm{CH}_{2}\right)_{\mathrm{n}}-$ & 0.40 \\
$-\mathrm{CH}_{2} \mathrm{CO}-$ & 0.26 \\
carbonyl & 1.8 \\
$-\mathrm{PO}-\mathrm{CH}_{2}$ & 0.41 \\
$-\mathrm{CH}_{2} \mathrm{~N}-$ & 0.30 \\
$\mathrm{~N}\left(\mathrm{CH}_{3}\right)_{3}$ & 0.62 \\
\hline \multicolumn{2}{c}{ 'Taken from Reference 83. See also } \\
Reference 6.
\end{tabular}

shown that the spin-lattice relaxation rate can be approximated by

$$
\frac{1}{T_{1}} \approx A \tau_{\|}+B \frac{1}{\omega_{0}^{2} \tau_{\perp}},
$$

with the first term dominating the observed temperature dependence, and the second term dominating the observed frequency dependence. On the basis of proton $T_{1}$ studies Feigenson \& Chan (17) have estimated the following ranges of correlation times for the liquid crystalline phase

$$
\tau_{\|} \approx 10^{-9}-2 \times 10^{-10} \mathrm{sec}
$$

and

$$
\tau_{\perp} \geq 10^{-7} \mathrm{sec} .
$$

The origin of the motions bearing these correlation times is not obvious, and accordingly several different motional models have emerged. Generally speaking, the slower time, $\tau_{\perp}$, has been neglected entirely and the faster time, $\tau_{\|}$, has been related to rotation about the long axis of the molccule in terms of various schemes for trans-gauche bond isomerization along the chains. Since the spin-lattice relaxation times are dominated by the more rapid motions, modulations of the dipolar interactions by motions occurring on the timescale of $\tau_{\|}$are the most effective $T_{1}$ processes. Kimmich \& Peters (85) have calculated ${ }^{1} \mathrm{H}$ relaxation rates for the gel phase based on a kink diffusion model, while Gent \& Prestegard (86) have calculated both ${ }^{1} \mathrm{H}$ and ${ }^{13} \mathrm{C}$ relaxation rates for the liquid crystalline phase by considering the effects of both kink formation and annihilation and isolated trans-gauche isomerizations, occurring on timescales of $10^{-10}$ and $10^{-7}$ seconds, respectively. Both of these calculations are in good agreement with experimental results, and are much more 
satisfactory than previous calculations which only included isomerization about individual bonds $(87-89)$.

Chan and co-workers $(17,18)$ were the first to consider, in detail, motions related to $\tau_{\perp}$. Originally, these workers interpreted the two timescales, $\tau_{\| 1}$ and $\tau_{\perp}$, as the correlation times for the motion of the interproton magnetic dipolar vector parallel to and perpendicular to the chain axis, respectively. More recently, they have refined their interpretation of these correlation times, relating $\tau_{\perp}$ more specifically to the rate of chain reorientation and $\tau_{\|}$to the rate of chain isomerization, where chain isomerization occurs by a kink diffusion process (44).

The diffusion of a kink along the phospholipid acyl chain can occur through a set of $\delta$-coupled trans-gauche isomerizations $(44,85)$, as is illustrated in Figure 3. Using the notation of Flory (90), a kink is denoted by $k_{n}^{ \pm}$, where the subscript denotes the carbon atom closest to the acyl end of the chain, such that the first trans-gauche rotation in the kink occurs around the bond between carbons $n$ and $n+1$, and the superscript denotes the sense of that bond rotation. The $\delta$-coupled rotations transform an even kink into another even kink but with opposite sense. Kink diffusion corresponds to interconversion among even or odd kinks with alternating sense, e.g.

$$
k_{n-4}^{+} \rightleftharpoons k_{n-2}^{-} \rightleftharpoons k_{n}^{+} \rightleftharpoons k_{n+2}^{-} \rightleftharpoons k_{n+4}^{+} \text {. }
$$

There are therefore four subsets of kinks, with interconversion among the subsets only possible either 1 . through a totally eclipsed conformation, which is energetically
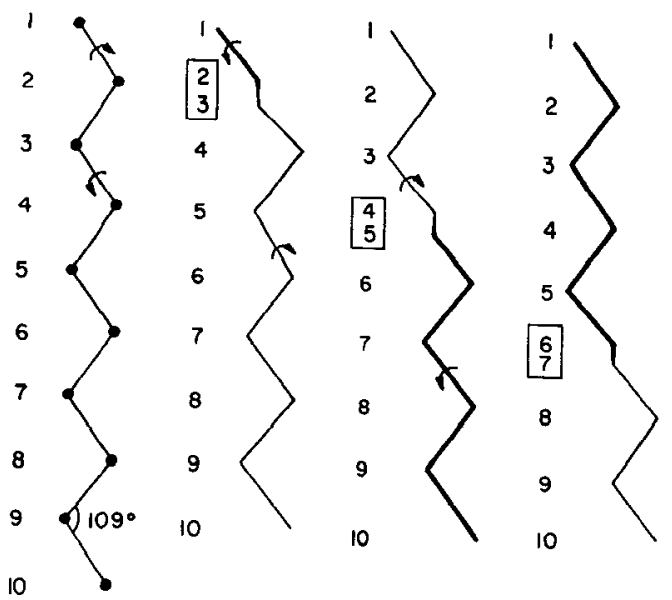

Figure 3 Schematic illustration of $\delta$-coupled trans-gauche \pm rotations around a kink leading to kink diffusion. The arrows indicate the sense of the rotations keeping the highernumbered carbon fixed, and the particular sequence illustrated corresponds to, from left to right: all-trans $\rightarrow k_{1}^{+} \rightarrow k_{3}^{-} \rightarrow k_{5}^{+}$. The carbon atoms are numbered, and the pair of methylene segments which are oriented off axis with respect to the chain are indicated by the rectangle around the carbon numbers. It is evident that this kink diffusion sequence will affect every methylene segment along the chain. Taken from Reference 44. 
unfavorable, or 2. by kink annihilation to form the all-trans chain followed by formation of a kink of different sense or at a new position. Kink formation is most likely to initiate at the terminal end of the chain where there is the least steric hindrance. Once formed, the kink can diffuse up and down the chain until it is ultimately dissipated at the terminal methyl group. The correlation time, $\tau_{\|}$, is the timescale associated with the departure and return of a kink to a given segment. This view of the segmental motion is supported by the positive gradient of ${ }^{13} \mathrm{C},{ }^{1} \mathrm{H}$, and ${ }^{2} \mathrm{H}$ relaxation times toward the terminal end of the chain $(6,8,24,81,83)$, which acts as a kink sink. By applying a simple one-dimensional diffusion model, where the energy of activation for $\delta$-coupled rotations is $3-4 \mathrm{kcal} / \mathrm{mole}(76)$, $\tau_{\|}$can be estimated to be $10^{-10} \mathrm{sec}(44)$, which is the order of magnitude inferred from the $T_{1}$ data.

The kink diffusion model also suggests that the frequency with which a kink appears in a certain position should be sensitive to the number of available positions for the kink. Consequently, $T_{1}$ should be sensitive to chainlength. In agreement with this prediction, Kainosho et al (91) have found that $T_{1}$ decreases as the chainlength increases from a total of 12 to 18 carbons.

If the correlation time, $\tau_{\perp}$, for multilayers arises from fluctuations of the tilt of the chain with respect to the bilayer normal, then $\tau_{\perp}^{-1}$ measures the rate at which the chain traverses the angular range determined by the distribution function $g(\alpha)$ (44). In general, one expects $\tau_{\perp}$ and the fluctuations in $\alpha$ to exhibit a complex dependence on the properties of the bilayer, particularly since the chain reorientation could be partly molecular and partly cooperative in origin. The measured $\tau_{\perp}$ really then reflects the most effective combination of timescales and amplitudes of the chain reorientation for the spin-lattice relaxation process.

Finally, we comment on the tendency of some workers to correlate chain flexibility, as determined by the magnetic resonance methods discussed above, and mobility as inferred from $T_{1}$ measurements. This correlation is misleading if not fallacious. The mobility of the system pertains to how $\tau_{\|}$varies with the position of the methylene segment, while chain flexibility is a measure of the orientational order and its variation along the chain. The Hamiltonian for a motionally restricted system can be formally written as

$$
\mathscr{H}=\overline{\mathscr{H}}+(\mathscr{H}(t)-\overline{\mathscr{H}}) .
$$

Orientational order is inferred from $\mathscr{H}$, whereas the motional information is contained in $\mathscr{H}(t)-\overline{\mathscr{H}}$; more specifically, the spin-lattice relaxation rate $1 / T_{1}$ is of the order of $(\mathscr{H}(t)-\overline{\mathscr{H}})^{2} \tau_{\|}$. It has been customary to assume in the interpretation of $T_{1}$ data of membrane systems that $\breve{\mathscr{H}}$ is zero, and this, of course, is incorrect for a motionally restricted system. Accordingly the interpretation of $T_{1}$ gradients is not as straightforward as once assumed.

\section{Lateral Diffusion}

Although the exchange of phospholipid molecules between the two halves of the bilayer is quite slow [on the order of days $(92,93)]$, diffusion parallel to the plane of the bilayer is rapid and should, in principle, be measurable with NMR methods. 
In practice, however, lateral diffusion rates of membrane components are difficult to measure directly with NMR, because conventional techniques for studying translational diffusion, such as pulsed magnetic field gradient spin echoes (94), are severely limited by the residual static dipolar interactions present in membrane systems. Magnetic field gradient techniques have been used to measure lateral diffusion coefficients of cubic phases and lamellar phases aligned between glass plates and oriented at the "magic angle," where the manifestations of the static dipolar interactions are significantly reduced $(95,96)$. With these methods, values of $D \approx 2 \times$ $10^{-6} \mathrm{~cm}^{2} / \mathrm{sec}$ and $1 \times 10^{-7} \mathrm{~cm}^{2} / \mathrm{sec}$ have been determined for single alkyl chain lipids and lecithins, respectively. The method of stimulated spin echoes (97) can also be used in systems where $T_{2} \ll T_{1}$, and using this method Roberts (98) has measured diffusion coefficients for amphiphilic molecules in both hexagonal and lamellar mesophases.

Another difficulty associated with the measurement of lateral diffusion rates by NMR methods is the possibility of interference from residual normal water, when these measurements are made in $\mathrm{D}_{2} \mathrm{O}$. Even small amounts of normal water can contribute significantly to the measured value of $D$. The lateral diffusion coefficient of liquid crystalline phase phospholipids has been estimated on the basis of ESR spin label and other studies to be $D \approx 10^{-8} \mathrm{~cm}^{2} / \mathrm{sec}(73,99,100)$. The discrepancy between this value and that determined by NMR methods may be due, in part, to the interference of water in the latter measurements.

Recently, Cullis (72) used ${ }^{31} \mathrm{P}$ NMR to estimate the lateral diffusion coefficient of lipids in sonicated vesicles as well. Cullis assumed that the ${ }^{31} \mathrm{P}$ linewidths observed in vesicles were controlled only by vesicle tumbling and lateral diffusion, and separated out the contributions from vesicle tumbling by increasing the solvent viscosity. At temperatures above the phase transition, the diffusion coefficient was determined to be $D \approx 2 \times 10^{-8} \mathrm{~cm}^{2} / \mathrm{sec}$, which is identical to that determined for multilayers. Below the phase transition temperature, Cullis obtained a twenty-fold decrease in the lateral diffusion coefficient. These values for the lateral diffusion coefficient in the gel and liquid crystalline phases are in good agreement with recent fluorescence bleaching-recovery experiments where values of $D \approx 5 \times 10^{-8} \mathrm{~cm}^{2} / \mathrm{sec}$ and $5 \times 10^{-10} \mathrm{~cm}^{2} / \mathrm{sec}$ have been measured for the two phases, respectively (101). Lee et al (102) have also reported the determination of lateral diffusion coefficients for lipids in sonicated vesicles. These workers estimated values of $D$ on the basis of intermolecular contributions to the proton linewidths and spin-lattice relaxation rates, but Kroon et al (76) later invalidated their data and analysis.

\section{HEAD GROUP ORIENTATION AND DYNAMICS}

The structural and dynamical properties of aqueous dispersions of phospholipids are strongly influenced by the nature of the polar head group in these molecules. Inasmuch as the head groups interact among themselves and with water, these interactions, along with the van der Waals interactions between the hydrophobic acyl chains, dictate the thermodynamic properties, and control the structure and 
phase behavior of phospholipid-water dispersions. The head group presumably also plays an important role in modulating the activity of the interfacial region, which may have biological implications. For these reasons, much recent effort has been devoted toward elucidating the organization of this region of the bilayer at the molecular level.

\section{Head Group Orientation}

Direct measures of the order in the polar regions of phospholipid multilayer systems have recently been obtained from analysis of ${ }^{31} \mathrm{P}$ chemical shift anisotropy and deuterium quadrupolar splittings $(23,29-34)$. Phosphorus-31 NMR has been previously used to investigate the phospholipid head group $(6,8,103-106)$, but most ${ }^{31} \mathrm{P}$ experiments have been performed on sonicated vesicles, under which conditions the chemical shift anisotropy is rotationally averaged to zero, and hence most of the structural information that may be gleaned from the ${ }^{31} \mathrm{P}$ shielding tensor is lost. Some early experiments were performed on multilamellar dispersions $(28,105)$, but for these systems either high proton-dccoupling powers or large magnetic fields $(35,103,104)$ are necessary to reduce significantly the effects of ${ }^{31} \mathrm{P}-{ }^{1} \mathrm{H}$ dipolar interactions.

Kohler \& Klein (33) have reported ${ }^{31} \mathrm{P}$ static shielding tensors for powdered samples of several phospholipids and related organophosphates. The spectra were obtained under the conditions of strong proton-decoupling in conventional FID experiments and in cross polarization (107) experiments. The two techniques resulted in essentially identical spectra, but the cross polarization experiment allowed acquisition of transients at a rate six times faster than the conventional experiment. Kohler \& Klein's results (33) clearly indicate that the static ${ }^{31} \mathrm{P}$ shielding tensor is nonaxial, a conclusion that conflicts with the previous measurements by McLanghlin et al (29), who found the shielding tensors to be axially symmetric. However, McLanghlin et al obtained their spectra without proton-decoupling, and it is possible that the ${ }^{1} \mathrm{H}$ dipolar broadening obscures the true chemical shift anisotropy pattern in their experiments.

Both Kohler \& Klein $(33,34)$ and Seelig and co-workers $(30-32)$ have examined the chemical shift anisotropy of aqueous multilamellar dispersion of phospholipids in some detail. With the addition of water, the chemical shift tensor becomes axially symmetric and is narrowed from its static dispersion of about $230 \mathrm{ppm}$ to approximately $50 \mathrm{ppm}$. Both ${ }^{31} \mathrm{P}$ and ${ }^{2} \mathrm{H}$ NMR experiments on oriented bilayers confirm that the axis of symmetry is the bilayer normal $(23,35)$. Griffin (108) has systematically studied the ${ }^{31} \mathrm{P}$ chemical shift anisotropy as a function of water content at $15^{\circ} \mathrm{C}$ and found that the reduction of the shielding tensor anisotropy is complete after the addition of approximately $10 \%$ water by weight. This corresponds to complete hydration of the head group after the addition of four or five water molecules, which is consistent with previous ${ }^{1} \mathrm{H}$ NMR measurements (109).

Measurements of the chemical shift anisotropy as a function of temperature (30) show that warming the bilayer through the phase transition further reduces the chemical shift anisotropy, but that the anisotropy stays nearly constant in the 
temperature range a few degrees below the thermal pretransition $(110-112)$ to the main phase transition. The chemical shift anisotropy in this temperature range for a $50 \%$ lecithin/water mixture is approximately the same as for samples containing $10 \%$ water at a temperature of $15^{\circ} \mathrm{C}$. On the basis of these observations, both Gally et al (30) and Griffin (108) have questioned the validity of interpreting the calorimetric pretransition solely in terms of a change in the state of motion of the head group (113). In this regard, McLaughlin (35) and Cullis \& DeKruijff $(114,115)$ have proposed that there is a change in the rotational motion of the entire lipid molecule about its long axis near the temperature of the pretransition.

By using the values of the static shielding tensor determined by Kohler \& Klein (33), Niederberger \& Seelig (31) have expressed the residual ${ }^{31} \mathrm{P}$ chemical shift anisotropy, $\sigma_{a}$, observed for aqueous dispersions of phospholipids in terms of $S_{11}$ and $S_{33}$, the order parameters of the principal axis connecting the two esterified and two nonesterified oxygens, respectively.

$$
\sigma_{a}(\text { in } \mathrm{ppm})=-56 S_{11}+133 S_{33}
$$

Because of the nonaxially symmetric static shielding tensor of the ${ }^{31} \mathrm{P}$ nucleus, two order parameters are necessary to describe the orientational order of the phosphate group. Although $S_{11}$ and $S_{33}$ cannot be determined without additional information, Scelig and co-workers (31) have used the results of their ${ }^{2} \mathrm{H}$ NMR experiments (30) to predict that the axis connecting the nonesterified oxygen atoms is oriented parallel to the plane of the bilayer. They further predicted that the choline group has a flexible temperature-dependent conformation, with increasing temperature stabilizing a structure where the trimethyl ammonium group is in the vicinity of the phosphate. This picture is consistent with the results of solution studies of choline and phosphatidylcholine, where the $\mathrm{C}-\mathrm{C}$ bond between the choline methylene groups was found to be in the gauche conformation exclusively for phosphatidylcholine, and predominantly for choline (116-118). Seelig and co-workers subsequently made a more quantitative interpretation of ${ }^{31} \mathrm{P}$ chemical shift anisotropy and ${ }^{2} \mathrm{H}$ quadrupolar data, and concluded that the choline dipole lies parallel to the plane of the bilayer (119). This boomerang-shaped conformation (Figure 4) for the head group is in accord with both high resolution X-ray and neutron diffraction studies on single crystals of phospholipids and phospholipid homologues (120-123).

Several dynamical models have been proposed for the head group motion in phospholipids. Kohler \& Klein (34) have suggested a model based on averaging of the ${ }^{31} \mathrm{P}$ shielding tensor observed in multilamellar dispersions, while Seelig and co-workers $(32,119)$ have proposed a model based on both ${ }^{31} \mathrm{P}$ and ${ }^{2} \mathrm{H}$ NMR data. Both of these models adequately account for the present experimental data, and a choice between them is not possible on the basis of this information alone. An earlier model for the dynamical motion proposed by McLaughlin et al (29) is not acceptable, because it is based on the assumption that the static ${ }^{31} \mathrm{P}$ shielding tensor is axially symmetric.

Kohler \& Klein (34) found that two different schemes of simple uncoupled rotations could successfully simulate the observed narrowing of the ${ }^{31} \mathrm{P}$ shielding tensor. The first combination of head group motions (A) consists of a fast rotation about 


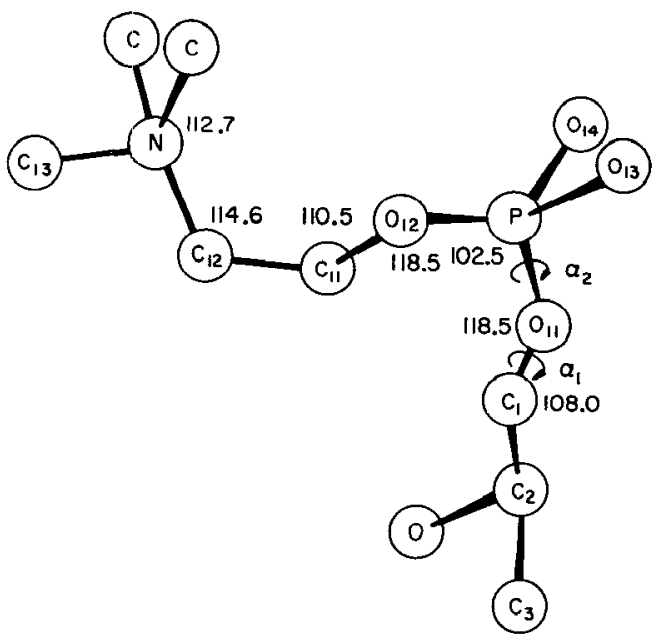

Figure 4 Conformation of the head group in glycerylphosphorylcholine. Taken from Reference 119.

the bond $\mathrm{P}-\mathrm{O}_{11}$, followed by a slower rotation about the glycerol $\mathrm{C}_{1}-\mathrm{C}_{2}$ bond, followed by a still slower overall rotation about the long molecular axis. The second possible combination of motions (B) includes only two rotations, a fast rotation about $\mathrm{P}-\mathrm{O}_{11}$, followed by a slower rotation about the long molecular axis. By including a wobble about the long axis of the phospholipid, Kohler \& Klein were also able to reproduce the observed temperature dependence of the residual chemical shift anisotropy.

The Seelig-Gally model (32) differs from the Kohler-Klein model (34) in several respects. Seelig \& Gally assume a conformation about bonds $\mathrm{C}_{1}-\mathrm{O}_{11}$ and $\mathrm{P}-\mathrm{O}_{11}$ and allow torsional angles $\alpha_{1}$ and $\alpha_{2}$ to jump between specified values. Using these angles, the shielding tensor is transformed to a reference frame rotating about bond $\mathrm{C}_{1}-\mathrm{C}_{2}$, and the tensor is averaged. $\mathrm{A}$ further averaging is introduced by using the order parameter determined with ${ }^{2} \mathrm{H}$ NMR as a measure of the motion about $\mathrm{C}_{1}-\mathrm{C}_{2}$. This model is most like Kohler \& Klein's model B with the wobble, but differs in that model $\mathrm{B}$ proposes free rotation about $\mathrm{P}-\mathrm{O}_{11}$ and assumes no rotation about $C_{1}-C_{2}$, while the Seelig-Gally model assumes free rotation about $C_{1}-C_{2}$ and conformational jumps about $\mathrm{P}-\mathrm{O}_{11}$.

\section{Head Group Dynamics}

Proton NMR studies on multilamellar dispersions of phospholipids have shown that the head groups also undergo restricted anisotropic motion $(17,18)$. In multilayer systems, only the choline methyl resonances are sharp enough to be clearly resolved, while the head group methylene proton resonances are much broader. Feigenson \& Chan (17) have interpreted the choline methyl proton spin-lattice 
relaxation rates in terms of two correlation times: $\tau_{\|}=1 \times 10^{-10}-5 \times 10^{-11} \mathrm{sec}$ for reorientation about the rotor axis and $\tau_{\perp}>4 \times 10^{-7} \mathrm{sec}$ for off axis excursions. The value of the faster correlation time, $\tau_{\|}$, is in agreement with the correlation time of $\tau_{c}=1.9 \times 10^{-11}-3.3 \times 10^{-11} \mathrm{sec}$ determined from deuterium $T_{1}$ measurements (30). The activation energy for reorientation calculated from the ${ }^{2} \mathbf{H}$ NMR experiments is also in accord with previous proton $(15,124)$ and deuterium $(23) T_{1}$ measurements for the choline methyl group in sonicated bilayer vesicles. With ${ }^{13} \mathrm{C} N M R$, the individual resonances of the head group methyl and methylene carbons have been resolved for both sonicated vesicles and solutions of phospholipids (79-83). The ${ }^{13} \mathrm{C}$ spin-lattice relaxation rates, shown in Table 1 , indicate that a positive mobility gradient exists from the glycerol moiety towards the choline methyl group.

Phosphorus-31 NMR has also been used extensively to investigate the head group region of sonicated vesicles (3-11). The bulk of these studies skillfully exploited the chemical shift differences of the phosphate moiety in different head groups to monitor the spatial distribution of different lipids in the highly strained mixed lipid vesicle systems. More recently, Martin and co-workers $(125,126)$ have reported ${ }^{31} \mathrm{P}$ spin-lattice relaxation rates and nuclear Overhauser effects (NOE) for a number of different phospholipid systems. These workers obtained results that suggest that the most probable orientation for the choline group in the vesicle is in a plane parallel to the bilayer surface with the nine methyl protons being, on the average, $3.2 \AA$ from the phosphorus atom. In a subsequent investigation, using mixed vesicles of phosphatidylcholine and phosphatidylethanolamine (127), they concluded that the ${ }^{31} \mathrm{P}_{-}{ }^{1} \mathrm{H}$ interactions modulating the NOE were largely intermolecular in origin.

\section{FUTURE PROSPECTS}

Despite the impressive number of NMR studies already performed on phospholipid bilayer systems, there is still valuable information to be gained with magnetic resonance techniques. Recent ${ }^{2} \mathrm{H}$ and ${ }^{31} \mathrm{P}$ NMR studies of membrane systems dramatically illustrate this point. The development of new experimental techniques also extends the range of possible investigations. Cross polarization and multiple pulse techniques have already been applied successfully to the investigation of phospholipid systems $(18,27,33)$, and newer methods such as interferometric NMR spectroscopy $(128,129)$ may also have application for the study of membranes. In addition, more work is necessary to confirm the validity of the various motional models for phospholipids in bilayers, and more importantly, to assess the importance of the motions themselves on the structure and function of natural membrane systems.

A number of NMR studies have already been performed on model systems composed of lipid/cholesterol and lipid/protein mixtures, which more closely simulate natural membranes. Apart from the phospholipids, cholesterol is the most common low molecular weight component of natural membrane systems, and there have been many attempts to determine its effect on phospholipid structure and mobility. Despite these efforts, the detailed nature of cholesterol-phospholipid 
interactions remains unclear. Deuterium NMR studies have shown that acyl chain order parameters are larger for cholesterol/phospholipid mixtures than in pure phospholipid dispersions $(14,23)$. On the basis of this information, Oldfield (E. Oldfield, private communication) has attempted to explain the effects of cholesterol using the Petersen-Chan model for phospholipid motion (44). He finds that the experimental order parameters are adequately modeled by allowing decreased chain reorientation upon the addition of cholesterol. Godici \& Lansberger (130) have measured ${ }^{13} \mathrm{C} T_{1}$ 's and linewidths for phospholipids in the presence of the cholesterol, and also suggested that cholesterol decreases the long range swinging of the acyl chains. The effects of protein on phospholipid motion have also been investigated with ${ }^{13} \mathrm{C}$ NMR using recombinant lipoprotein/lipid dispersions (131133). These studies suggest that lipid-protein interactions are primarily hydrophobic in nature rather than ionic $(131,133)$, and that lipids interacting with protein arc somewhat less mobile than in pure lipid systems (132).

NMR studies have also been conducted on biological membrane systems, where the dynamical state and structure of the phospholipids depend on the details of the lipid, lipopolysaccharide, and protein composition. Such biological systems as sarcoplasmic reticulum, erythrocyte, chloroplast, and mitochondrial membranes, as well as nerve myelin and rabbit sciatic nerve, have all been studied by NMR (8). Because of the complexity of these systems, most interpretations of the NMR spectra have been made by direct analogy to spectra of similar model systems. More recently, biosynthetic labeling techniques have allowed detailed investigations of natural membranes with ${ }^{13} \mathrm{C}$ and ${ }^{2} \mathrm{H}$ NMR. Activation energies calculated from ${ }^{13} \mathrm{C}$ spinlattice relaxation times obtained for yeast cells indicate that the molecular mobility of the membrane is similar to that of model systems (134). This suggests that the perturbation of the bilayer by protein is relatively small in this system. In a ${ }^{2} \mathrm{H}$ NMR study of rat liver mitochondrial membranes, Arvidson et al (135) have found that the deuterium quadrupolar splitting of the choline methyl group is much less than in lamellar dispersions prepared from the lipid extract. They suggest that this difference is the result of a difference in the orientation of the head group in the natural and extracted lipids. Brown et al (136) have obtained ${ }^{1} \mathrm{H}$ NMR spectra of retinal rod outer segment (ROS) disk membranes and liposomes of purified extracted ROS phospholipids. The spectra of these two preparations are similar and suggest that the phospholipids are organized into at least two lateral domains, which differ in the fluidity of the acyl chains of the phospholipids in the respective regions. These workers further suggest that the lipid domains directly interacting with the rhodopsin molecules are the most fluid, and that the protein does not inhibit large amplitude segmental or self-diffusive motions of these lipids.

These examples of recent work clearly indicate that the trends for future significant work lie toward more complex model systems, which more closely simulate biomembranes, or the real membranes themselves. There is every reason to expect that sustained efforts in these directions, augmented by the detailed knowledge of membrane structure and dynamics already acquired through studies of simple model systems, will ultimately result in a more complete understanding of the complex processes involved in membrane-associated phenomena. 


\section{ACKNOWLEDGEMENT}

The authors gratefully acknowledge support by Grant No. 22432 from the National Institute of General Medical Sciences, US Public Health Service. This is contribution No. 5724 from the Division of Chemistry and Chemical Engineering.

\section{Literature Cited}

1. Hubbell, W. L., McConnell, H. M. 1971. J. Am. Chem. Soc. 93:314-26

2. Jost, P., Waggoner, A. S., Griffin, O. H. 1971. In Structure and Function of Biological Membranes, ed. L. Rothfield, Chap. 3. New York: Academic. $486 \mathrm{pp}$.

3. Chapman, D., Salsbury, N. J. 1970. Recent Progr. Surf. Sci. 3:121-68

4. Chapman, D. 1973. Protides Biol. Fluids Proc. Colloq., ed. H. Peeters, $21: 165-73$. Oxford: Pergamon. 677 pp.

5. Chapman, D., Oldfield, E. 1974. Methods Enzymol. (B). 32:198 211

6. Lee, A. G., Birdsall, N. J. M., Metcalf, J. C. 1974. Methods Membr. Biol. 2:1-156

7. Podo, F. 1975. Biochimie 57:461-69

8. James, T. L. 1975. Nuclear Magnetic Resonance in Biochemistry, Chap. 8. New York: Academic. 413 pp.

9. Wennerström, H., Lindblom, G. 1977. Q. Rev. Biophys. 10:67-96

10. Tiddy, G. J. T. 1975. Nucl. Magn. Reson. (Spec. Period. Rep.) 4:233-52

11. Tiddy, G. J. T. 1977. Nucl. Magn. Reson. (Spec. Period. Rep.) 6:207-32

12. Luzzati, D. 1968. Biol. Membr. 1:71121

13. Charvolin, J., Rigny, P. 1973. J. Chem. Phys. 58:3999-4008

14. Ulmius, J., Wennerström, H., Lindblom, G., Arvidson, G. 1975. Biochim. Biophys. Acta 389: 197-202

15. Horwitz, A. F., Horsley, W. J., Klein, M. P. 1972. Proc. Natl. Acad. Sci. USA $69: 590-93$

16. Hemminga, M. A., Berendsen, H. J. C. 1972. J. Magn. Reson. 8:133-43

17. Feigenson, G. W., Chan, S. I. 1974. $J$. Am. Chem. Soc. 96:1312-19

18. Seiter, C. H. A., Chan, S. I. 1973. J. Am. Chem. Soc. 95:7541-53

19. Seelig, J., Seelig, A. 1974. Biochem. Biophys. Res. Commun. 57:406-11

20. Seelig, J., Seelig, A. 1974. Biochemistry 13:4839-45

21. Seelig, J., Niederberger, W. 1974. J. Am. Chem. Soc. 96:2069-72

22. Seelig, J., Niederberger, W. 1974. Biochemistry 13:1585-88
23. Stockton, G. W., Polnaszek, C. F., Leitch, L. C., Tullock, A. P., Smith, I. P. C. 1974. Biochem. Biophys. Res. Commun. 60:844-50

24. Stockton, G. W., Polnaszek, C. F., Tullock, A. P., Hasan, F., Smith, I. P. C. 1976. Biochemistry 15:954-66

25. Longmuir, K. J., Dahlquist, F. W. 1976. Proc. Natl. Acad. Sci. USA 73:2716-19

26. Gent, M. P. N., Armitage, I. M., Prestegard, J. H. 1976. J. Am. Chem. Soc. 98:3749-55

27. Urbina, J., Waugh, J. S. 1974. Proc. Natl. Acad. Sci. USA 71:5062-67

28. Sheetz, M. P., Chan, S. I. 1972. Biochemistry 11:4573-81

29. McLaughlin, A. C., Cullis, P. R., Berden, J. A., Richards, R. E. 1975. J. Magn. Reson. 20:146-65

30. Gally, H.-U., Niederberger, W., Seelig, J. 1975. Biochemistry 14:3647-52

31. Niederberger, W., Seelig, J. 1976. $J$. Am. Chem. Soc. 98:3704-6

32. Seelig, J., Gally, H.-U. 1976. Biochemistry 15:5199-5204

33. Kohler, S. J., Klein, M. P. 1976., Biochemistry 15:967-73

34. Kohler, S. J., Klein, M. P. 1977. Biochemistry 16:519-26

35. McLaughlin, A. C., Cullis, P. R., Hemminga, M. A., Hoult, D. I., Radda, G. K., Ritchie, G. A., Seely, P. J., Richards, R. E. 1975. FEBS Lett. 57:213-18

36. Abragam, A. 1961. The Principles of Nuclear Magnetism, Chap. 4. Oxford: Clarendon. 599 pp.

37. Mely, B., Charvolin, J., Keller, P. 1975. Chem. Phys. Lipids 15:161-73

38. Gaffney, B. J., McConnell, H. M. 1974. J. Magn. Reson. 16:1-28

39. Perchold, W. 1968. Kolloid Z. Z. Polym. 228:1-38

40. Träuble, H. 1971. J. Membr. Biol. 4:193-208

41. Marčelja, S. 1974. Biochim. Biophys. Acta 367: 165-76

42. Schindler, H., Seelig, J. 1975. Biochemistry $14: 2883-87$

43. Ukleja, P., Pirs, J., Doane, J. W. 1976. Phys. Rev. A 14:414-23 
44. Petersen, N. O., Chan, S. I. 1977. Biochemistry 16:2556-67

45. Jackson, M. B. 1976. Biochemistry 15:2555-61

46. Lawson, K. D., Flautt, T. J. 1965. $J$. Phys. Chem. 69:4256-68

47. Lawson, K. D., Flautt, T. J. 1968. J. Phys. Chem. 72:2066-74

48. Kaufman, S., Steim, J. M., Gibbs, J. H. 1970. Nature 225:743-44

49. Hansen, J. R., Lawson, K. D. 1970. Nature 225:542-43

50. Chan, S. I., Feigenson, G. W., Seiter, C. H. A. 1971. Nature 231:110-12

51. Charvolin, J., Rigny, P. 1971. J. Magn. Reson. 4:40-46

52. Chan, S. I., Seiter, C. H. A., Feigenson, G. W. 1971. Biochem. Biophys. Res. Commun. 46: 1488-92

53. Wennerström, H. 1973. Chem. Phys. Lett. 18:41-44

54. Bloom, M., Burnell, E. E., Valic, M. I,, Weeks, G. 1975. Chem. Phys. Lipids $14: 107-12$

55. Bloom, M., Burnell, E. E., Roeder, S. B. W., Valic, M. I. 1977. J. Chem. Phys. 66:3012-20

56. Jeener, J., Broekaert, P. 1967. Phys. Rev. 157:232-40

57. Petersen, N. O., Kroon, P. A., Kainosho, M., Chan, S. I. 1975. Chem. Phys. Lipids 14:343-49

58. Anderson, P. W. 1954. J. Phys. Soc. Jpn. 9:316 39

59. Niederberger, W., Seelig, J. 1974. Ber. Bunsenges. Phys. Chem. 78:947-49

60. 'Birrell, G. B., Griffin, O. H. 1976. Arch. Biochem. Biophys. 172:455-62

61. Mendelson, R., Sunder, S., Bernstein, H. J. 1976. Biochim. Biophys. Acta 419:563-69

62. Gaber, B. P., Peticolas, W. L. 1976. Biochim. Biophys. Acta 465:260-74

63. Kawato, S., Kinosita, K., Ikegami, A. 1977. Biochemistry 16:2319-24

64. Finer, E. G., Flook, A. G., Hauser, H. 1972. Biochim. Biophys. Acta 260: $49-58$

65. Finer, E. G., Flook, A. G., Hauser, H. 1972. Biochim. Biophys. Acta 260: 59-69

66. Finer, E. G. 1974. J. Magn. Reson. $13: 76-86$

67. Wennerström, H., Ulmius, J. 1976. $J$. Magn. Reson. 23:431-35

68. Chan, S. I., Sheetz, M. P., Seiter, C. H. A., Feigenson, G. W., Hsu, M., Lau, A. 1973. Ann. NY Acad. Sci. 222:499-522

69. Lichtenberg, D., Petersen, N. O., Girardet, J.-L., Matsune, K., Kroon, P. A., Seiter, C. H. A., Feigenson,
G. W., Chan, S. I. 1975. Biochim. Biophys. Acta 382:10-21

70. Horwitz, A. F., Michaelson, D., Klein, M. P. 1973. Biochim. Biophys. Acta $298: 1-7$

71. Gutowsky, H. S., Pake, G. E. 1950. J. Chem. Phys. 18:162-70

72. Cullis, R. P. 1976. FEBS Lett. 70: 223-28

73. Edidin, M. 1974. Ann. Rev. Biophys. Bioeng. 3:179-201

74. Curatolo, W., Shipley, G. G., Small, D. M., Sears, B., Neuringer, L. J. 1977. J. Am. Chem. Soc. $99 ; 6771-72$

75. Kostelenik, R. J., Castellano, S. M. 1973. J. Magn. Reson. 9:291-95

76. Kroon, P. A., Kainosho, M., Chan, S. I. 1976. Biochim. Biophys. Acta 433:282-93

77. Chrzeszczyk, A., Wishnia, A., Springer, C. S 1977. Biochim. Biophys. Acta 470:161-69

78. Daycock, J. T., Dark, A., Chapman, D. 1971. Chem. Phys. Lipids 6:205-14

79. Levine, Y. K., Birdsall, N. J. M., Lee, A. G., Metcalf, J. C. 1972. Biochemistry $11: 1416-21$

80. Gent, M. P. N., Prestegard, J. H. 1974. Biochem. Biophys. Res. Commun. 58: 549-55

81. Lee, A. G., Birdsall, N. J. M., Metcalf, J. C. 1973. Chem. Br. 9:116-23

82. Sears, B. 1975. J. Membr. Biol. 30: $59-73$

83. Godici, P. E., Lansberger, F. R. 1974. Biochemistry 13:362-68

84. McLaughlin, A. C., Podo, F., Blasie, J. K. 1973. Biochim. Biophys. Acta $330 \div 109-21$

85. Kimmich, R., Peters, A. 1975. $J$. Magn. Reson. 19:144-65

86. Gent, M. P. N., Prestegard, J. H. 1977. J. Magn. Reson 25:243-62

87. Levine, Y. K., Parington, P., Roberts, G. C. K. 1973. Mol. Phys. 25:497-514

88. Levine, Y. K. 1973. J. Magn. Reson. $11: 421-25$

89. Levine, Y. K., Parington, P., Roberts, G. C. K., Birdsall, N. J. M., Lee, A. G., Metcalf, J. C. 1972. FEBS Lett. 23: 203-7

90. Flory, P. J. 1969. In Statistical Mechanics of Chain Molecules, p. 13. New York: Wiley-Interscience. 432 pp.

91. Kainosho, M., Kroon, P. A., Lawaczeck, R., Petersen, N. O., Chan, S. I. 1978. Chem. Phys. Lipids. In press

92. Kornberg, R. G., McConnell, H. M. 1971. Biochemistry 10:1111-20

93. Rothman, J. E., Dawidowicz, E. A. 1975. Biochemistry $14: 2809-16$ 
94. Tanner, J. E., Stejskal, E. O. 1968. J. Chem. Phys. 49:1768-77

95. Roeder, S. B. W., Burnell, E. E., Kuo A.-L., Wade, C. G. 1976. J. Chem. Phys. 64:1848-49

96. Lindblom, G., Wennerström, H. Int. Congr. Magn. Reson., Biol., 8th, St. Jovite, Quebec, Canada, Sept. 1976

97. Tar.ver, J. E. 1970. J. Chem. Phys. $52: 2523-26$

98. Roberts, R. T. 1973. Nature 242:348

99. Devaux, P., McConnell, H. M. 1972. J. Am. Chem. Soc. 94:4475-81

100. Träuble, H., Sackman, E. 1972. J. Am. Chem. Soc. $14: 4499-4510$

101. Wu, E.-S., Jacobson, K., Papahadjapoulos, D. 1977. Biochemistry 16: 3936-41

102. Lee, A. G., Birdsall, N. J. M., Metcalf, J. C. 1973. Biochemistry 12:1650-59

103. Davis, D. G. 1972. Biochem. Biophys. Res. Commun. 49:1492-97

104. Berden, J. A., Cullis, P. R., Hoult, D. I., McLaughlin, A. C., Radda, G. K., Richards, R. E. 1974, FEBS Lett. 46:55-58

105. Horwitz, A. F., Klein, M. P. 1972. J. Supramol. Struct. $1: 19-28$

106. Michaelson, D. M., Horwitz, A. F., Klein, M. P. 1974. Biochemistry 13: 2605-12

107. Pines, A., Gibby, M. G., Waugh, J. S. 1973. J. Chem. Phys. 59:569-90

108. Griffin, R. G. 1976. J. Am. Chem. Soc. $98: 851-53$

109. Veksli, S. J., Salsbury, N. J., Chapman, D. 1969. Biochim. Biophys. Acta 183: 434-46

110. Chapman, D., Williams, R. M., Ladbrook, B. D. 1967. Chem. Phys. Lipids $1: 445-75$

111. Ladbrook, B. D., Williams, R. M., Chapman, D. 1968. Biochim. Biophys. Acta 150:333-40

112. Hinz, H., Sturtevant, J. M. 1972. J. Biol. Chem. 247:6071-75

113. Steim, J. M. 1960. Adv. Chem. Ser. $84: 259-302$

114. Cullis, P. R., DeKruijff, B. 1976. Biochim. Biophys. Acta 426:433-46

115. Cullis, P. R., DeKruijff, B. 1976. Biochim. Biophys. Acta 436:523-40

116. Birdsall, N. J. M., Fecncy, J., Lee, A. G., Levine, Y. K., Metcalf, J. C. 1972. J. Chem. Soc. Perkin Trans. $2: 1441-45$
117. Dufourcq, J., Lussan, C. 1972. FEBS Lett. 26:35-38

118. Lichtenberg, D., Kroon, P. A., Chan, S. I. 1974. J. Am. Chem. Soc. 96: 5934-36

119. Seelig, J., Gally, H.-U., Wohlgemuth, R. 1977. Biochem. Biophys. Acta 467: $109-19$

120. Lesslauer, W., Cain, J. E., Blasie, J. K. 1972. Proc. Natl. Acad. Sci. USA 1972:1499-1503

121. Franks, N. P. 1976. J. Mol. Biol. $100: 345-58$

122. Worcester, D. L., Franks, N. P. 1976. J. Mol. Biol. 100:359-78

123. Worcester, D. L. 1976. Biol. Membr. $3: 1-45$

124. Lee, A. G., Birdsall, N. J. M., Levine, Y. K., Metcalf, J. C. 1972. Biochim. Biophys. Acta 255:43-56

125. Yeagle, P. L., Hutton, W. C., Huang, C.-H., Martin, R. B. 1975. Proc. Natl. Acad. Sci. USA 72:3477-81

126. Yeagle, P. L., Hutton, W. C., Martin, R. B. 1975. J. Am. Chem. Soc. 97: 7175-77

127. Yeagle, P. L., Hutton, W. C., Huang, C.-H., Martin, R. B. 1976. Biochemistry 15:2121-24

128. Stoll, M. E., Vega, A. J., Vaughan, R. W. 1977. Phys. Rev. A 16:1521-24

129. Stoll, M. E., Vega, A. J., Vaughan, R. W. 1977. J. Chem. Phys. 67:202938

130. Godici, P. E., Landsberger, F. R. 1975. Biochemistry 14:3927-33

131. Assman, G., Highet, R. J., Sokoloski, E. J., Brewer, H. B. 1974. Proc. Natl. Acad. Sci. USA $71: 3701-5$

132. Stoffel, W., Zierenberg, O., Tunggal, B. D., Schreiber, E. 1974. Z. Physiol. Chem. 355:1381-90

133. Hamilton, J.A., Talkowski, C., Childers, R. F., Williams, E., Allerhand, A., Cordes, E. H. 1974, J. Biol. Chem. 249:4872-78

134. London, R. E., Kollman, V. H., Matwiyoff, N. A. 1974. Biochemistry 14: 5492-5500

135. Arvidson, G., Lindblom, G., Drakenberg, T. 1975. FEBS Lett. 54:249-52

136. Brown, M. F., Miljanich, G. P., Dratz, E. A. 1977. Biochemistry 16:2640-48 
Annual Review of Physical Chemistry

Volume 29, 1978

\section{CONTENTS}

Chemical Bonding, Robert S. Mulliken

Light Energy Transduction in Visual Pigments and

BACTERIORHODOPSIN, Barry Honig

Information Theory Approach to Molecular Reaction Dynamics, R. D. Levine

Circular Dichroism SPectroscopy and the VacuUm Ultraviolet

Region, W. Curtis Johnson, Jr.

Inelastic Electron Tunneling Spectroscopy: A Probe of the Vibrational Structure of Surface Species, $W$. Henry Weinberg

Microcalorimetry of Biological Systems, B. George Barisas and Stanley J. Gill

NMR Relaxation Studies of Solute-Solvent Interactions, Robert G. Bryant

Photoelectrochemistry: Applications to Solar Energy Conversion, Arthur J. Nozik

Atom and Radical Recombination Reactions, Jürgen Troe

High Resolution Electron Microscopy of Crystal Defects and Surfaces, J. M. Cowley

Kinetic Processes on Metal Single-Crystal Surfaces, $R$. $J$. Madix and J. Benziger

NMR Studies of Membrane Structure and Dynamics, David F. Bocian and Sunney I. Chan

High Resolution NMR Studies of The Structure and Dynamics of tRNA In Solution, Dinshaw $J$. Patel

The Description of Chemical Bonding from Ab Initio Calculations, William A. Goddard III and Lawrence B. Harding

High Resolution, Solid State NMR, Robert $W$. Vaughan

Nonlinear Spectroscopy, Robert L. Swofford and A. C. Albrecht 421

Structures of Molecular Liquids, David Chandler 
Piezoelectricity, Pyroelectricity, and Ferroelectricity in OrganiC Materials, $R$. G. Kepler INDEXES

AUTHOR INDEX $\quad 559$

SUBJECT INDEX $\quad 582$

Cumulative Index of Contributing Authors, Volumes 25-29 597

Cumulative Index of Chapter Titles, Volumes 25-29 598 